\title{
Multi-Information Fusion-Based Hierarchical Power Generation-Side Protection System
}

\author{
Xianggen Yin ${ }^{1}$, Yikai Wang ${ }^{1, *}$, Jian Qiao ${ }^{1}$, Wen $\mathrm{Xu}^{1}{ }^{1}$, Xin Yin ${ }^{2}$, Lin Jiang ${ }^{2}$ and Wei $\mathrm{Xi}^{3}$ \\ 1 State Key Laboratory of Advanced Electromagnetic Engineering and Technology, Huazhong University of \\ Science and Technology, 1037 Luoyu Road, Wuhan 430074, China; xgyin@mail.hust.edu.cn (X.Y.); \\ d201980439@hust.edu.cn (J.Q.); 18810207012@163.com (W.X.) \\ 2 Department of Electrical Engineering and Electronics, The University of Liverpool, Liverpool L69 3BX, UK; \\ leoxinyin@hotmail.com (X.Y.); ljiang@liverpool.ac.uk (L.J.) \\ 3 Digital Grid Research Institute, China Southern Power Grid, Guangzhou 510663, China; xiwei@csg.cn \\ * Correspondence: d201880427@hust.edu.cn; Tel.: +86-151-2722-7626
}

Citation: Yin, X.; Wang, Y.; Qiao, J.; Xu, W.; Yin, X.; Jiang, L.; Xi, W. Multi-Information Fusion-Based Hierarchical Power Generation-Side Protection System. Energies 2021, 14, 327. https://doi.org/10.3390/en 14020327

Received: 25 October 2020 Accepted: 6 January 2021 Published: 8 January 2021

Publisher's Note: MDPI stays neutral with regard to jurisdictional clai$\mathrm{ms}$ in published maps and institutional affiliations.

Copyright: (C) 2021 by the authors. Licensee MDPI, Basel, Switzerland. This article is an open access article distributed under the terms and conditions of the Creative Commons Attribution (CC BY) license (https:// creativecommons.org/licenses/by/ $4.0 /)$.

\begin{abstract}
With renewable power sources and new topology structures being widely introduced into the power system, the current local information-based power generation-side protection cannot fully guarantee the protection performance and the safety coordination with the power grid. This paper proposes an improved hierarchical protection system on the power generation-side. The proposed system takes advantage of the fusion of multi-information provided by the system, station and local layers. The system layer provides the information such as system voltage control and power regulation demand, so that the generation-side protection and control system can adapt to the system operation mode and power regulation demand. The station layer realizes the coordination of the protection principle and action strategy among the related units through information interaction with the power grid, the automatic control system and the local layer protection. The local layer introduces the condition monitoring information and more abundant protection information to enhance the protection performance and master the generation units' safety condition. To illustrate the hierarchical protection system construction method and actual application mode, the multiinformation fusion-based comprehensive local layer protection method and the multi-generators information fusion-based hierarchical protection method are taken as examples. Case analysis shows that the proposed methods can reflect more slight internal fault forms and can adaptively determine the protection action characteristic and tripping strategy according to the system operating conditions and other generators' fault conditions, which effectively improve the protection sensitivity and coordination capability. To provide reference and inspiration for follow-up research, the hierarchical protection system construction mode, communication technology and research approaches of new protection methods are further pointed out.
\end{abstract}

Keywords: power generation-side; multi-information fusion; hierarchical protection system; system layer; station layer; local layer

\section{Introduction}

In order to satisfy green and low-carbon development, the power system has introduced large-scale renewable power sources, new power transmission technologies and new topology structures. As the main power supporting source, the security problems of the conventional large power generators and their coordinated operation with the power grid are becoming increasingly prominent. The current generation-side protection only uses local information. However, it cannot master the generation units' safety condition, and cooperation between the two is lacking. It cannot accomplish the coordination between source and grid and lacks the information interaction between the protection system and the automatic monitoring devices. Since the protection and control modes are selected improperly and cooperate ineffectively, many accidents have been caused such as generator 
damage or even system blackouts [1-7], which has greatly harmed the safe operation of the power system. Therefore, it is urgent to enhance the safety defense ability of the power generation-side protection system.

In order to guarantee the protection performance and achieve the effective coordination of the generation-side protection with the system operation requirements, the current research mainly focuses on the principle improvement of the generator protection related to the network and its coordination with the automatic control system. The literature $[8,9]$ proposes an improved out-of-step protection method based on equal-area-criterion theory, which can more accurately discriminate the transient stability state and the out-of-step fault state. The literature [10] proposes a fault-current-based stator ground fault protection method which constructs the protection criterion and determines the generator tripping strategy according to the fault degree. In order to improve the regulation and control ability of the generator excitation regulation system and the automatic control system, the study in [11] standardizes the coordination relationship of the loss-of-excitation protection and the low excitation limitation. In addition, the literature [12] gives a coordination scheme of the over-speed protection and the high-frequency tripping measures of the automatic control system. All the above methods can improve the power support ability of the generation-side under fault conditions. However, due to lack of more abundant local fault information and appropriate information interaction between the generators and between the generation and grid sides, the protection performance cannot be further improved.

With the rapid development of communication technology and the application of the wide area measurement system [13-15], the hierarchical transmission network protection system has been widely concerned, studied and applied in some engineering. From this, a variety of new protection technologies have been developed. Among them, wide area current differential protection $[16,17]$ can achieve the effective backup protection coordination under system disturbance situations. Wide area back-up protection [18-20] can quickly accomplish the fault component identification, tripping decision and other functions through information interaction by the wide area communication network. Station area integrated protection [21,22] acquires the multi-information on the station layer at the same time, and the protection function coordination can be realized on the basis of completing each independent protection function. However, the multi-information fusionbased hierarchical protection technology is still lacking in the generation-side protection system, which restricts the safe operation of the main pieces of equipment in the power plants and their coordination with the power system under the modern complex power grid environment.

In order to overcome the protection performance limitations and the multi-generator protection coordination problems, on the basis of the existing local protection system, condition monitoring system and power control system, the hierarchical protection system is established through multi-information interaction and fusion among the above systems, the generation units and the power grid. This paper discusses the structure and function of the hierarchical power generation-side protection system. The local layer multiinformation fusion-based comprehensive protection method and the multi-generators information fusion-based hierarchical protection method are taken as examples to illustrate the protection principle construction method and the actual application mode. Finally, the key issues of how to complete the hierarchical power generation-side protection system are discussed. Compared with the current protection methods, the multi-information fusion-based hierarchical protection methods can successfully improve the protection sensitivity and can adaptively determine the protection action characteristic and tripping strategy according to the system operating conditions and other generators' fault conditions. They can effectively enhance the protection performance and provide reliable guarantee for the safe operation coordination of the generation and grid sides. 


\section{Structure and Function of the Hierarchical Power Generation-Side Protection System}

The goal of the multi-information fusion-based hierarchical power generation-side protection system is to enhance the protection performance and satisfy the security and coordinated operation requirements between the generation and grid sides. The hierarchical protection system combines the information from the condition monitoring system, power generation control system, local protection system and power grid dispatch and control system to accomplish the information interaction and fusion. On this basis, the relatively independent design and function of the current generation-side protection system can be changed.

Figure 1 shows the structure diagram of the multi-information fusion-based hierarchical power generation-side protection system. It is composed of a local layer, station layer and system layer.

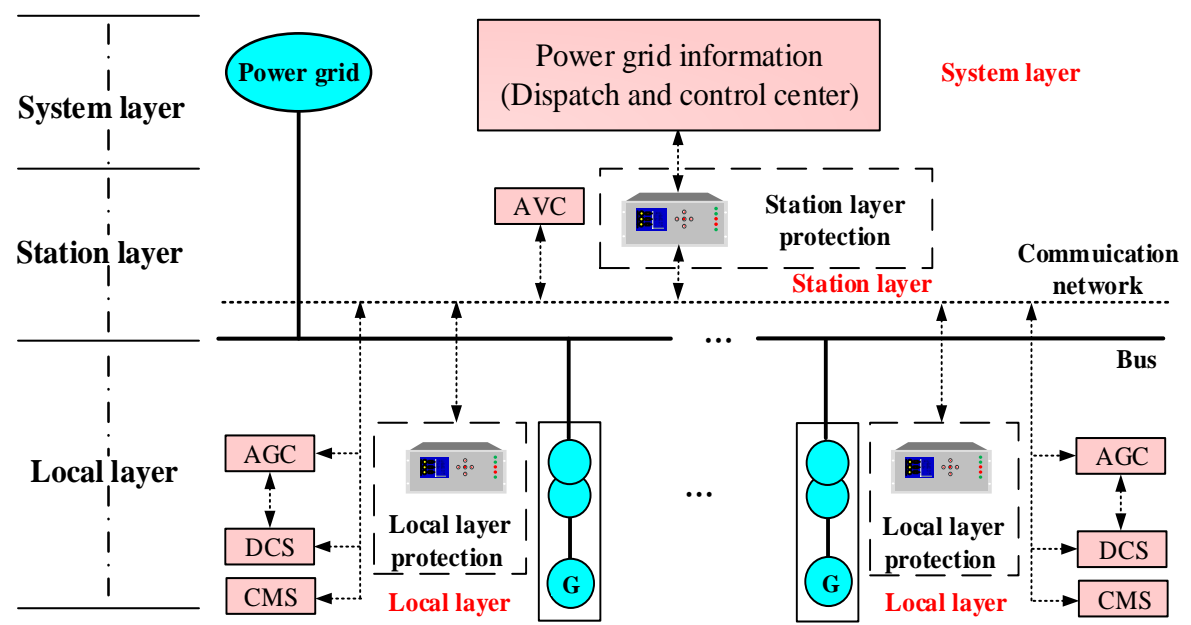

Figure 1. Structure diagram of the multi-information fusion-based hierarchical power generation-side protection system.

As for the local layer, it is composed of the local generation units' protection devices to achieve the local protection function. However, its protection information source is not limited to the local measurement data, it also includes the fused information from other generation units' protection, automatic voltage control system (AVC), automatic generation control system (AGC), distributed control system (DCS) and condition monitoring system (CMS) through the communication network supported by the station layer. In the hierarchical protection system, the local layer protection can not only satisfy the rapidity and reliability requirements of the local protection function, but also use the condition information and control information to master the healthy condition of the generation units and improve the protection performance. In addition, the local layer protection undertakes the safety protection instructions assigned by the station layer, such as warning, tripping the generator, adjusting the generation output, etc.

The station layer is a new layer in the generation-side protection system on the basis of the existing protection devices. It can provide a transmission platform for information interaction and fusion between the generation units and the power grid, serving as the information management center of the whole protection system. It can satisfy the local layer protection information requirements and realize the station layer protection function. More importantly, it can enhance the performance of the grid-related generation-side protection, and better adapt to the cooperative and safe operation between the generation and grid sides. In addition, the station layer also accomplishes the unified voltage control and power output dispatching of the generators in the power plant according to the instructions from the system layer. The existing dispatch and control center adopts the "one to one" power dispatching mode, which is directly targeted at the specific generator. Due to lack 
of information interaction, the healthy condition of the generator cannot be mastered by the dispatch and control center. In the situation that the generator output is adjusted but is being made to enter an unhealthy operation condition, if the protection acts and the generator is quickly tripped, the system power shortage will become worse. However, if the protection does not act in time, the generator may be damaged. After introducing the station layer, the health condition constraints of the generators and the safety operation requirements of the power grid can be fully reflected. Thus, the dispatching and controlling strategy can effectively meet the real-time safety demand of the whole system.

The system layer is composed of the power grid dispatch and control center. It can monitor and grasp the voltage level of each node in the system and the power supply demand of the users. Through integrating the information from the whole network, it assigns the voltage regulation, power dispatching and other instructions which can meet the requirements of the whole power grid to the station layer. It also summarizes and analyzes the operation condition and protection information of the power grid-side and sends them to the generation-side. Then the station layer can attain the goal of remote information interaction, unified control and collaborative protection between the generation and grid sides.

The multi-information fusion-based hierarchical power generation-side protection system does not change the basic configuration of the existing protection devices and automatic monitoring devices. It only needs to add the communication network and set up the station layer protection device to complete the basic structure of the protection system, which is easily accomplished. The hierarchical protection system can make full use of the multi-information fusion and protection layering, which can enrich the protection information source, satisfy the protection coordination with the system operating environment and guarantee the security defense ability.

\section{Several Examples of the Hierarchical Generation-Side Protection System Construction Methods}

The existing generation-side protection system is constructed with local protection information. Due to lack of information fusion with CMS, the protection information is not complete enough to fully master the real-time security condition of the corresponding generation units. Moreover, since the protection information is not abundant enough, the protection performance cannot be further improved. In addition, since information interaction between the generation units and between the generation and grid sides cannot be achieved, the existing protection methods and action strategies are unable to attain the coordination among the protection units under the fault forms which affect multi-generators. In this section, the multi-information fusion-based comprehensive local layer protection construction method and the multi-generators information fusion-based hierarchical protection construction method are proposed as examples to illustrate the hierarchical protection construction method and the application mode.

\subsection{Multi-Information Fusion Based Comprehensive Local Layer Protection}

In order to solve the problem that the existing local information-based generation-side protection system cannot fully master the real-time security condition and cannot further enhance the protection performance, the multi-information fusion-based hierarchical protection system is used to improve the local layer protection principles. The relevant information such as electrical, mechanical and condition monitoring information which can reflect the safety condition of the generation units is introduced to enhance the protection sensitivity. In this section, the comprehensive single transverse differential protection criterion is taken as an example to illustrate the multi-information fusion-based comprehensive local layer protection construction method.

\subsubsection{Review of the Existing Single Transverse Differential Protection}

For large generators, their internal structures are complex and their operation conditions are changeable. Thus, the internal fault types are various and the fault forms are 
complex. For the slight turn-to-turn faults, due to the short-circuit current not being large, the protection sensitivity is usually low [23]. However, if the protection fixed value is blindly lowered to improve the protection sensitivity, the unbalanced current under external faults may lead to protection maloperation, causing unnecessary generator tripping and endangering the safe and stable operation of the power system.

The existing single transverse differential protection for the large generators usually uses the phase current braking criterion [24]. The braking criterion can prevent protection maloperation. However, the existing research $[25,26]$ shows that the changing rules are quite different between the phase current and the transverse differential unbalanced current, and they cannot establish a direct relationship. The protection method cannot avoid the unbalanced current effectively under different operation modes, which can easily cause protection maloperation. In addition, since the phase current braking criterion is used, the braking current is relatively large under internal faults. Thus, the protection sensitivity is reduced. Therefore, it is necessary to integrate other protection information which can fully reflect the transverse differential unbalanced current to improve the existing protection braking criterion.

\subsubsection{Air Gap Electromotive Force Braking Criterion for Single Transverse Differential Protection}

In order to solve the problem that the widely used phase current braking criterion cannot effectively reflect the transverse differential unbalanced current, the generation mechanism of the transverse differential unbalanced current is analyzed to find the exact electric quantity which can fully reflect it. Then a novel protection criterion is constructed.

Due to various reasons such as design, manufacturing, installation and operation, the internal structure of a generator cannot be absolutely symmetrical, which makes the inductance parameters (including self-inductance and mutual inductance) of each branch winding different. Thus, the imbalance of the main air gap flux is caused, and the imbalance of the stator winding electromotive force is caused. On this basis, the unbalanced current is generated on the connecting line of the neutral points $\left(\mathrm{O}_{1}\right.$ and $\left.\mathrm{O}_{2}\right)$; its value is related to the internal structure asymmetry degree and the main air gap flux strength. The schematic of the multi-branches generator stator windings is shown in Figure 2.

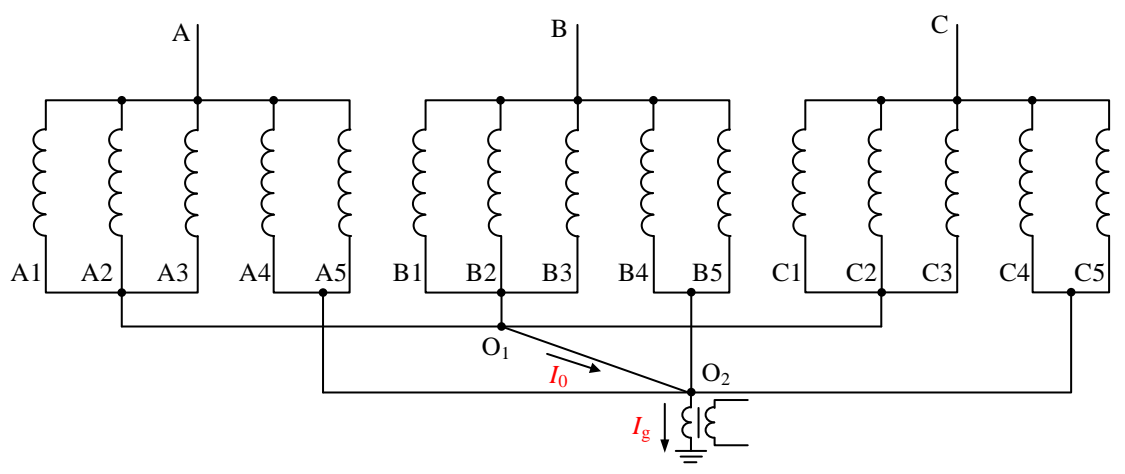

Figure 2. Schematic of the multi-branch generator stator windings.

Large generators usually use the small current grounding mode, and the low-voltage windings of the step-up transformer are usually connected by the delta type. Thus, the value of the ground current flowing into the earth from the neutral point $I_{\mathrm{g}}$ is very small. Compared with the value of the transverse differential unbalanced current $I_{0}$, it can be ignored. Based on this, in Figure 2, the parallel branches in the same phase which are connected at the same neutral point are equivalent to a single branch, and the equivalent circuit diagram is shown in Figure 3. 


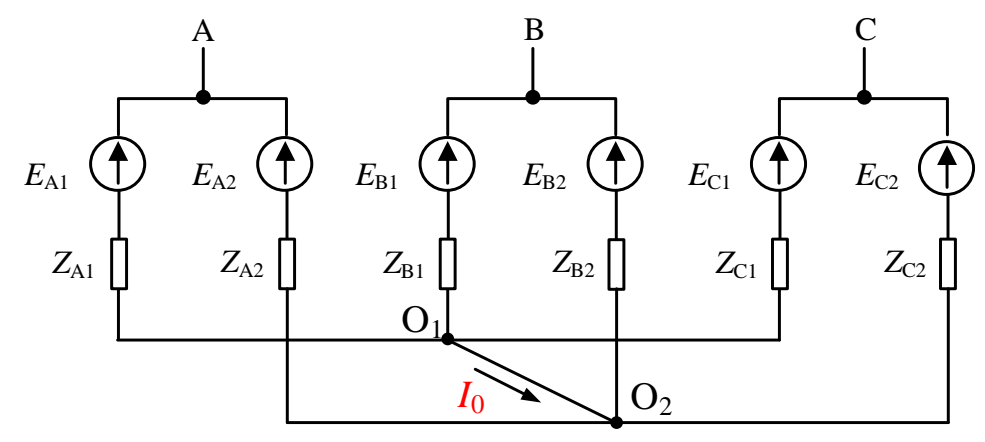

Figure 3. Equivalent circuit of the generator stator windings.

In Figure $3, E_{\mathrm{A} 1}, E_{\mathrm{A} 2}, E_{\mathrm{B} 1}, E_{\mathrm{B} 2}, E_{\mathrm{C} 1}, E_{\mathrm{C} 2}$ represent the comprehensive equivalent electromotive force of each branch and $Z_{\mathrm{A} 1}, Z_{\mathrm{A} 2}, Z_{\mathrm{B} 1}, Z_{\mathrm{B} 2}, Z_{\mathrm{C} 1}, Z_{\mathrm{C} 2}$ represent the comprehensive equivalent internal impedance of each branch. Since the transverse differential unbalanced current $I_{0}$ has the same property as the zero-sequence current, the three-phase branches connected at the same neutral point can be further combined into an equivalent branch, as shown in Figure 4.

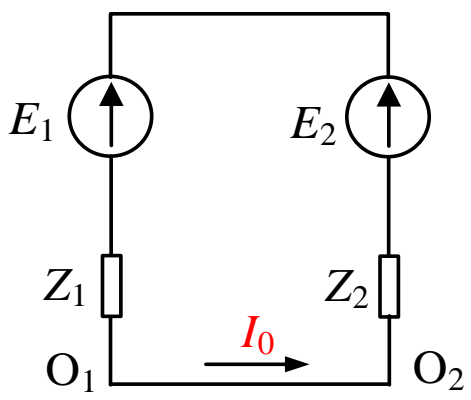

Figure 4. Simplified equivalent circuit of the generator stator windings.

In Figure $4, E_{1}$ and $E_{2}$ are the equivalent induced electromotive forces, reflecting the asymmetric degree of the three-phase induced electromotive force. $Z_{1}$ and $Z_{2}$ are the equivalent internal impedances. When the equivalent induced electromotive forces are not equal, the transverse differential unbalanced current on the neutral point line is produced as

$$
I_{0}=\frac{\left|E_{1}-E_{2}\right|}{Z_{1}+Z_{2}}=\frac{\Delta E}{Z_{1}+Z_{2}}
$$

where $\Delta E$ is the unbalanced induction electromotive force of the stator windings. Since it is generated by the alternations of the main air-gap flux with time, then

$$
\Delta E=4.44 f N k_{\mathrm{N} 1} \Delta \Phi_{\mathrm{m}}
$$

where $f$ is the power frequency, $N$ is the stator winding turns, $k_{\mathrm{N} 1}$ is the winding coefficient, and $\Delta \Phi_{\mathrm{m}}$ is the unbalanced main air gap flux of the stator windings which satisfies the following relationship

$$
\Phi_{\mathrm{m}} \propto L I
$$

where $L$ is the comprehensive equivalent inductance of the generator windings, and $I$ is the comprehensive equivalent current of the generator stator and rotor. Thus, the relationship between the unbalanced main air gap flux $\Delta \Phi_{\mathrm{m}}$ and the main air gap flux $\Phi_{\mathrm{m}}$ can be expressed as

$$
\Delta \Phi_{\mathrm{m}} \propto \frac{\Delta L}{L} \Phi_{\mathrm{m}}
$$


where $\Delta L$ means the winding inductance parameter difference of every branch, it reflects the asymmetry degree of the generator internal structure.

Once a generator is manufactured, its internal structure asymmetry remains unchanged. Combined with Equations (1)-(4), it can be analyzed that the transverse differential unbalanced current $I_{0}$ is proportional to the main air-gap flux amplitude $\Phi_{\mathrm{m}}$. According to the electric machinery theory, the main air-gap flux amplitude $\Phi_{\mathrm{m}}$ is proportional to the air gap electromotive force $E_{\delta}$. Thus, $E_{\delta}$ can be introduced to construct the protection criterion to monitor the change of the main air-gap flux. In addition, the monitoring result can be taken as the assistant criterion of the single transverse differential protection. The corresponding protection criterion is

$$
\begin{cases}I_{0}>I_{0 n} & E_{\delta} \leq E_{\delta n} \\ I_{0}>\frac{E_{\delta}}{E_{\delta n}} I_{0 n} & E_{\delta}>E_{\delta n}\end{cases}
$$

In Equation (5), $I_{0}$ is the measured transverse differential current, $E_{\delta}$ is the measured air gap electromotive force; the fixed value of $E_{\delta n}$ is set as the lowest air gap electromotive force when the generator operates normally and the fixed value of $I_{0 n}$ is set as the corresponding unbalanced current under the lowest air gap electromotive force. Among them, $E_{\delta}$ cannot be directly measured, it can be calculated by the following formula

$$
\dot{E}_{\delta}=\dot{U}_{g}+\dot{I}_{g}\left(R_{a}+j X_{\sigma}\right)
$$

where $R_{a}$ and $X_{\sigma}$ are the leakage resistance and the leakage reactance of the generator stator windings, respectively, they are the inherent parameters of the generator. $\dot{U}_{g}$ and $\dot{I}_{g}$ are the terminal voltage and current of the generator respectively, which can be measured by potential transformers and current transformers.

\subsubsection{Comprehensive Criterion for the Hierarchical Single Transverse Differential Protection}

The existing phase current braking criterion of the single transverse differential protection can effectively prevent the protection maloperation when external fault occurs. However, at the same time, the protection sensitivity under internal faults is reduced. As for the air gap electromotive force braking criterion, when internal turn-to-turn faults occur, due to the values of the voltage and current at the generator terminal generally do not have obvious change, the air gap electromotive force will not change. However, since the transverse differential current increases, the protection can act correctly. Even in some internal fault cases that the current at the generator terminal increases obviously, since the air gap electromotive force is also related to the terminal voltage and its fluctuation amplitude is generally small, the amplitude of the air gap electromotive force can be limited to a certain extent, and it can be prevented from entering the braking area.

In order to improve the performance of the local layer protection, based on the hierarchical protection system, the multi-information fusion-based method is used to construct the comprehensive protection criterion by combining the proposed air gap electromotive force braking criterion with the existing phase current braking criterion. Furthermore, the logical solution of the comprehensive protection criterion is shown in Figure 5.

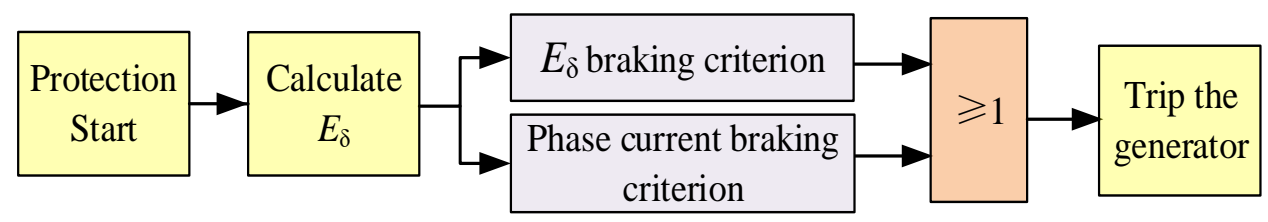

Figure 5. Logical solution of the hierarchical single transverse differential protection comprehensive criterion. 
The two criterions adopt the "or door" mode. When any criterion satisfies the action situation, the comprehensive single transverse differential protection will act. The two criterions can play to their respective advantages. The air gap electromotive force braking criterion can enlarge the protection scope and enhance the protection sensitivity for the internal small turn-to-turn faults, and the phase current braking criterion can prevent the protection maloperation when an external fault occurs. Thus, the protection performance can be effectively improved.

\subsubsection{Simulation Analysis of Protection Action Conditions under Generator Internal Faults}

In order to verify the correctness of the above theoretical analysis and the effectiveness of the proposed protection scheme, the relevant simulation analysis is carried out to test the transverse differential protection action conditions under internal faults. As for the large generator internal faults, the fault forms are large in number, and the electromagnetic transient relationship is very complex. Since the generator models in commonly used power system simulation software (such as ATP, EMTP, EMTDC, MATLAB, etc.) are packaged, the transient simulation calculation for internal faults cannot be carried out. Thus, the relevant simulation analysis is carried out based on the "Large hydro-generator internal fault simulation, main protection analysis and design system" developed by our research team. The detailed derivation and introduction of the model used in this software are presented in the literature $[27,28]$. The software system has been successfully applied to the internal fault simulation of the Three-Gorges hydropower plant and other large hydrogenerators. The experimental results and operating experience show that the simulation results can meet the engineering requirements.

To verify the viewpoint that the phase current cannot reflect the transverse differential unbalanced current, but the air gap electromotive force can, the $800 \mathrm{MW}$ generator of a hydropower plant is taken as an example, and the relationships of the transverse differential unbalanced current with the phase current and the air gap electromotive force during normal operation and external short circuit faults are simulated. Set the following simulation conditions: rated voltage and rated power factor with $10 \%, 40 \%, 70 \%, 100 \%$ load; the rated voltage and rated current with the power factors are $0.8,0.7$ and 0.6 , respectively; the rated current and rated power factor with the terminal voltage is $0.1,0.3,0.5$, 0.7, 0.9 times the rated voltage; The three-phase short-circuit fault current at the generator terminal is $10 \%, 40 \%, 70 \%$ and $100 \%$ of the rated current, respectively. For each condition, the transverse differential current $I_{0}$, the terminal voltage $U_{g}$ and the terminal phase current $I_{g}$ are measured, and the air gap electromotive force $E_{\delta}$ is calculated. The relation diagrams of $I_{0}$ with $E_{\delta}$ and $I_{0}$ with $I_{g}$ are shown in Figure 6.

The simulation results show that there is a linear relationship between $I_{0}$ and $E_{\delta}$ under both the normal operation conditions and the external fault conditions. However, there is no clear relationship between $I_{0}$ and $I_{g}$. The two are approximately linear only in the cases of three-phase short-circuit faults at the generator terminal corresponding to the data points in the circle of the Figure $6 \mathrm{~b}$. Therefore, it can be proved that the air gap electromotive force can reflect the transverse differential unbalanced current more effectively.

According to the winding structure of the case generator stator, the software generates the possible internal short-circuit fault set. Among them, "slot internal faults (SIF)" refers to the short circuit faults caused by insulation damage of the upper and lower coils in the same slot, and "terminal internal faults (TIF)" refers to the short circuit faults caused by insulation damage at the intersection of the coil terminals. According to the generator parameters and the simulation results, the parameters of the protection action curve can be calculated. The internal fault set and the corresponding protection action results are shown in Table 1. 


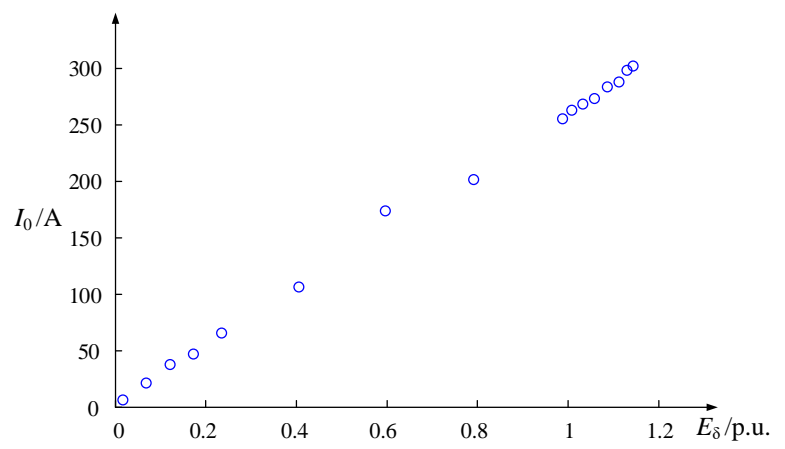

(a)

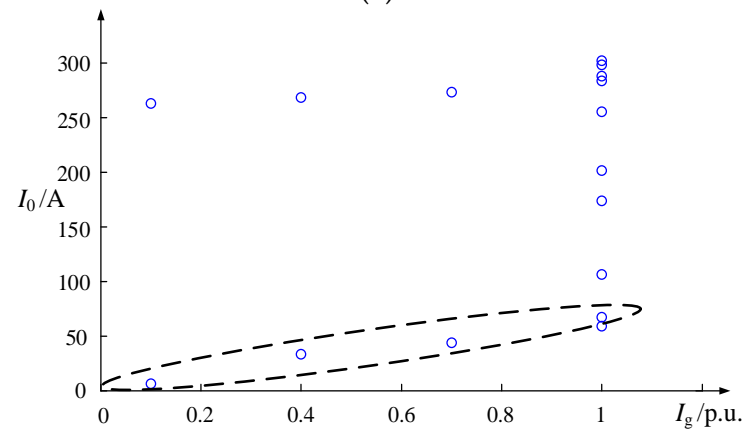

(b)

Figure 6. Simulation results of the generator transverse differential unbalanced current: (a) the relation diagram of the transverse differential unbalanced current with the air gap electromotive force; (b) the relation diagram of the transverse differential unbalanced current with the phase current.

Table 1. Statistical results of the generator internal fault set and the protection action condition results.

\begin{tabular}{|c|c|c|c|c|c|c|c|c|c|c|c|c|}
\hline & \multicolumn{4}{|c|}{ Internal Fault Set } & \multicolumn{4}{|c|}{ Comprehensive Protection Criterion } & \multicolumn{4}{|c|}{ Existing Protection Criterion } \\
\hline $\begin{array}{c}\text { Fault } \\
\text { Location }\end{array}$ & $\begin{array}{l}\text { Same } \\
\text { Branch }\end{array}$ & $\begin{array}{l}\text { Different } \\
\text { Branch }\end{array}$ & $\begin{array}{l}\text { Different } \\
\text { Phase }\end{array}$ & Total & $\begin{array}{l}\text { Same } \\
\text { Branch }\end{array}$ & $\begin{array}{l}\text { Different } \\
\text { Branch }\end{array}$ & $\begin{array}{l}\text { Different } \\
\text { Phase }\end{array}$ & Total & $\begin{array}{l}\text { Same } \\
\text { Branch }\end{array}$ & $\begin{array}{l}\text { Different } \\
\text { Branch }\end{array}$ & $\begin{array}{l}\text { Different } \\
\text { Phase }\end{array}$ & Total \\
\hline SIF & 432 & 48 & 360 & 840 & 428 & 48 & 360 & 836 & 420 & 48 & 360 & 828 \\
\hline TIF & 768 & 3792 & 11,400 & 15,960 & 765 & 3768 & 11,376 & 15,909 & 761 & 3723 & 11,342 & 15,826 \\
\hline Total & 1200 & 3840 & 11,760 & 16,800 & 1193 & 3816 & 11,736 & 16,745 & 1181 & 3771 & 11,702 & 16,654 \\
\hline
\end{tabular}

In order to compare the protection performance of the existing phase current braking criterion and the proposed comprehensive protection criterion, the action results of the two protection criterions are listed in Table 1 . By analyzing the statistical results, it can be seen that there are 16,800 forms of possible internal faults for the case generator. For the existing phase current braking protection criterion, the protection can act correctly under 16,654 forms of fault conditions. However, the comprehensive protection criterion can correctly act under 16,745 forms of fault conditions, which can supplement 91 kinds of faults compared with the existing protection criterion. These fault events are the slight internal faults for which the fault features are not obvious. However, since the large hydropower generator has a complex internal structure, these kind of fault forms have higher occurrence probability. If not removed in time, their long-term existence will also burn out the stator insulation and cause more serious faults. By introducing the air gap electromotive force braking criterion, the multi-information fusion-based comprehensive criterion of the single transverse differential protection can effectively reduce the protection action setting value under internal faults and the protection sensitivity is enhanced with the same braking ability under external faults. Thus, the protection performance is successfully improved. 


\subsection{Multi-Generators Information Fusion Based Hierarchical Protectio}

The existing generation-side protection system only uses the local protection information, and the information interaction between generation units and interaction between source and grid cannot be achieved. However, as for the fault forms which involve multiple generation units, they cannot be effectively reflected. By introducing the idea of hierarchical protection, the station layer protection is added on the basis of the original local layer protection. In this way, the protection can adaptively determine the action characteristic and tripping strategy according to the system operating situations and other generators' fault conditions. Through the coordination of the station layer, the local protection performance, the system control mode and the protection security defense ability can be advanced. In this section, the hierarchical out-of-step protection is taken as an example to illustrate the hierarchical protection construction method among different protection layers based on multi-generators information fusion.

\subsubsection{Review of the Existing Out-of-Step Protection}

The three-component impedance characteristic out-of-step protection criterion is usually used for large generators, and the protection action strategy is determined according to the impedance trajectory variation law $[29,30]$. Existing out-of-step protection only uses the local information, lacking information interaction and protection cooperation between generators. In addition, the protection fixed value is usually set according to the single generator out-of-step fault condition shown in Figure 7. However, since the current large power plants usually adopt multi-generators connected at one bus structure to supply power to the external power grid, when multi-generators become out-of-step at the same time, there may be a variety of out-of-step fault forms. Under different out-of-step forms, the existing protection mode faces difficulties in the action characteristics and generator tripping strategies.

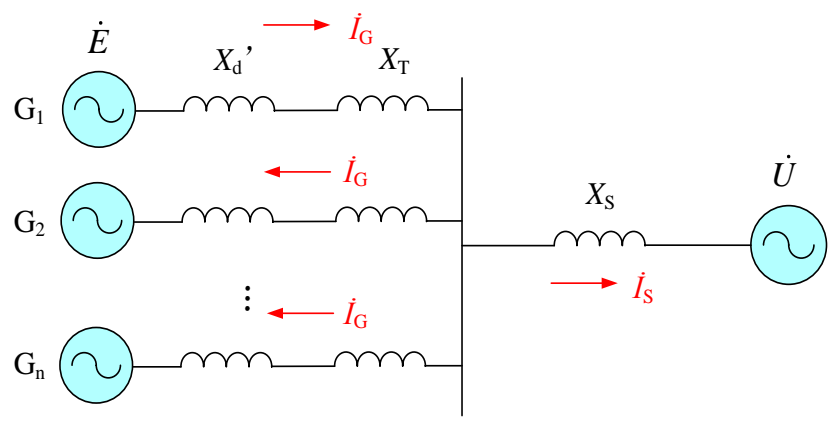

Figure 7. Schematic diagram of the single generator out-of-step condition.

As for the multi-generators connected at one bus out-of-step condition shown in Figure 8, for any out-of-step generator, the equivalent impedance of the system will be influenced by the oscillating current added from other generators. Thus, the measured impedance at the generator terminal will increase and its value is closely related to the operating mode of the power plant. Since the equivalent impedance value of the system is different in different out-of-step modes and different operating modes, the existing protection boundary cannot accurately identify the multi-generators out-of-step mode, which will result in protection maloperation or rejection. 


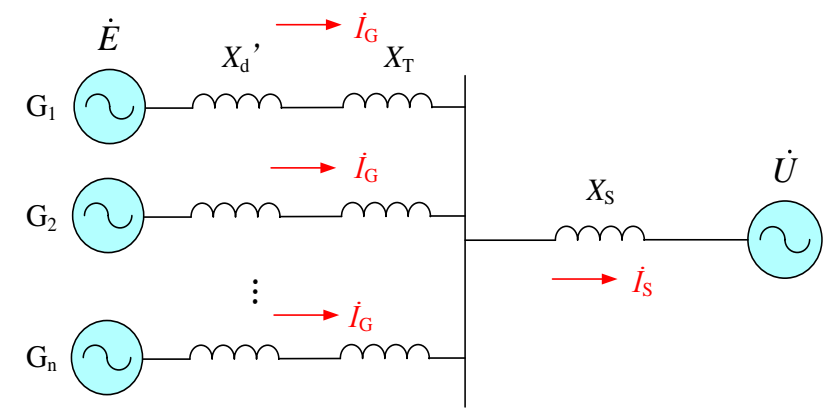

Figure 8. Schematic diagram of the multi-generators out-of-step condition.

In addition, for the existing out-of-step protection, each protection unit has the same protection fixed value. As long as the out-of-step oscillation between the generator and the external system is detected and the protection criterion meets action conditions, the protection will act and the corresponding generator will be tripped. However, this tripping strategy has no coordination with other connected generators. As for the multi-generators out-of-step condition, all the generators will be tripped at the same time, which harms the stability of the power system.

\subsubsection{Hierarchical Out-of-Step Protection}

In order to solve the protection principle defect and the action coordination problem under the multi-generators connected at one bus out-of-step conditions, combined with the existing three-component out-of-step protection criterion, the hierarchical out-of-step protection is constructed based on multi-generators information fusion. The out-of-step fault form can be identified by the cooperation of the station and local protection criterions, and the protection fixed value can be adaptively adjusted according to the operating mode of the power plant and the power system. The hierarchical out-of-step protection scheme is shown in Figure 9.

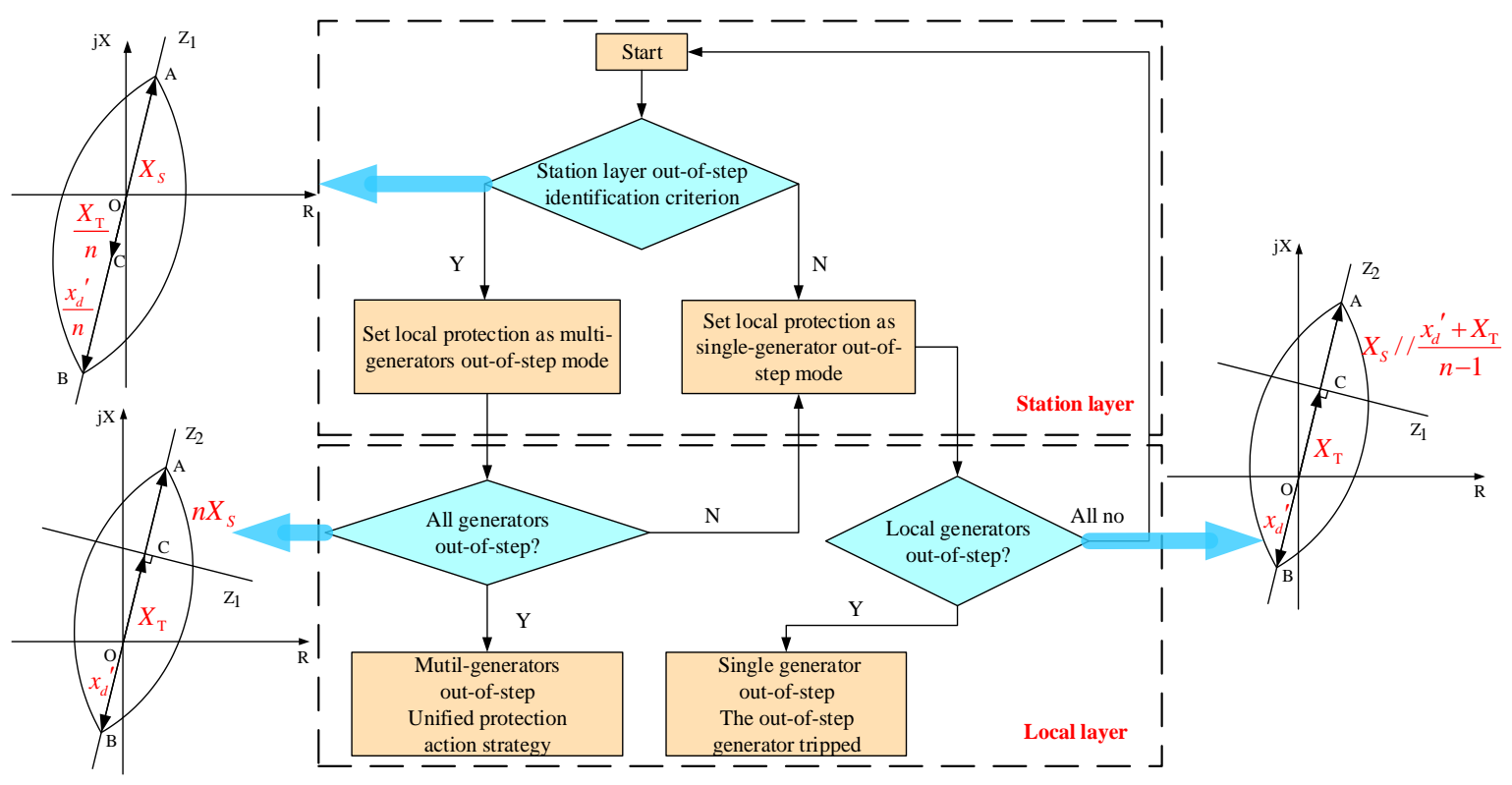

Figure 9. Hierarchical out-of-step protection.

The basic protection configuration, action characteristics and tripping strategy are set as follows:

(1) The basic protection configuration. In order to detect the multi-generators out-ofstep fault condition, the station layer out-of-step protection criterion is set at the 
bus. The local out-of-step protection criterion of each generator is retained to detect the single generator out-of-step fault. Through information interaction with the station layer protection, the local out-of-step protection criterion can adapt to different out-of-step modes and operating conditions.

(2) The protection action characteristics. The out-of-step protection action characteristics of the station layer and the local layer under different modes are shown in Figure 9. It is assumed that the parameters of all the generation units connected at the bus are the same. Among them, $x_{d}{ }^{\prime}$ means the subtransient reactance of the generator, $X_{\mathrm{T}}$ means the transformer reactance, $X_{S}$ means the equivalent system reactance, $n$ means the number of the generators operating in parallel. The out-of-step criterion of the station layer is set according to the impedance characteristics under multigenerators connected at one bus out-of-step fault condition. When it is judged that all the generators are out-of-step, the out-of-step criterion of the local layer is adaptively adjusted based on the enhancement effect of other generators. In the local layer, if the measured impedance trajectory of each local generator enters the multi-generators out-of-step action zone and meets the action condition, it can be ensured that the all the generators are out-of-step. Otherwise, the out-of-step criterion of the local layer should use the single generator out-of-step mode.

(3) The protection tripping strategy. When the protection judging result is the single generator out-of-step condition, the out-of-step generator is directly tripped at the appropriate time. When the judging result is multi-generators all in the out-ofstep condition, the instruction of the generators tripping by turns is issued by the station layer and executed by the local layer. Through information interaction with the grid-side, the tripping instructions are coordinated with the system stability control. After each generator is tripped, the station layer and local layer out-of-step protection need to measure the impedance trajectory again to determine the current out-of-step condition and tripping strategy. Until the system stability is restored, the protection returns.

\subsubsection{Simulation Analysis of Protection Action Conditions under Multi-Generators Out-of-Step Fault}

In order to verify the effectiveness of the hierarchical out-of-step protection scheme, the equivalent model shown in Figure 10 is constructed based on PSCAD/EMTDC software. Figure 10 shows that in large hydropower plant 1, 1\# generator-transformer group and 2\# generator-transformer group operate in parallel at the same bus M1. They are connected to the infinite system $S$ through the $500 \mathrm{kV}$ double-loop AC lines with the length of $200 \mathrm{~km}$. Among them, G2 and T2 are formed by equivalent generator-transformer units. They are under the same working conditions and with the same capacity. The actual number of the generator-transformer units depends on the specific simulation condition, and the maximum is three. In hydropower plant 2, G3 and T3 are formed by equivalent generator-transformer units with four parallel operating generator-transformer units. All the generator-transformer units in the model are equipped with prime mover, governor and excitation systems. The model can reproduce the operation condition and control process of the actual multi-generators system to some extent and meet the basic requirements of the multi-generators out-of-step fault simulation.

Four generator-transformer units are set to operate in parallel at bus M1. Before the fault occurs, each generator sends out the active power as $\mathrm{P}=600 \mathrm{MW}$ and reactive power as $\mathrm{Q}=167$ Mvar. Four generator-transformer units are set to operate in parallel at bus M2. Before the fault occurs, each generator sends out the active power as $P=400 \mathrm{MW}$ and reactive power as $\mathrm{Q}=116 \mathrm{Mvar}$. The three-phase short-circuit fault is set at $0.5 \mathrm{~s}$ on Line1 II near the bus M1, and the fault line is removed at $0.61 \mathrm{~s}$ by protection action. The power angle changes of the three equivalent generators are shown in Figure 11. 


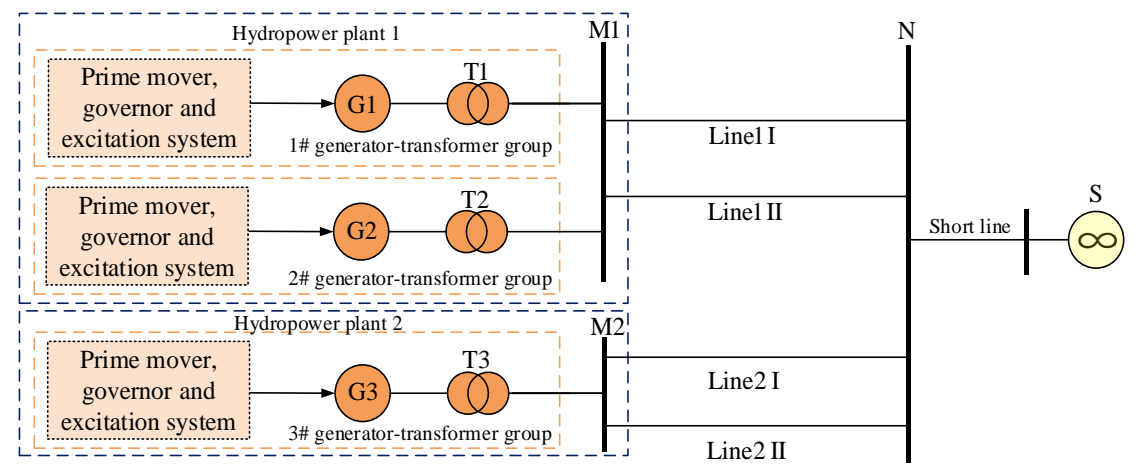

Figure 10. Schematic diagram of the out-of-step fault simulation model system.
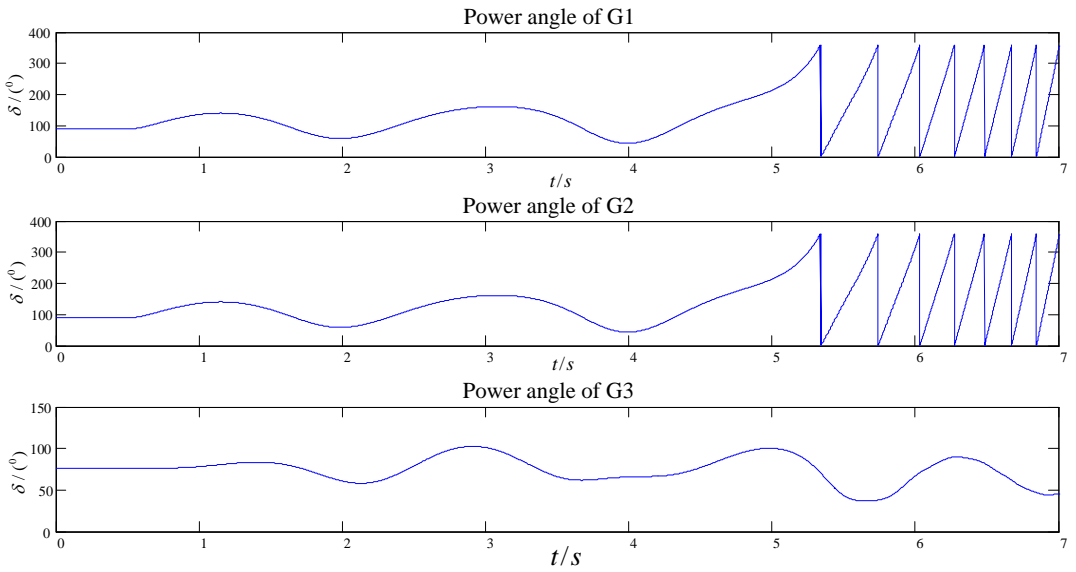

Figure 11. Power angle curve of the equivalent generators.

In Figure 11, after the fault is removed for a period of time, the equivalent generators G1 and G2 are out-of-step, and the equivalent generator G3 is oscillating synchronously. Therefore, the equivalent generators G1 and G2 constitute the situation of multi-generators connected at one bus out-of-step fault, and the equivalent generator G3 wobbles. According to the variation of the measured impedance trajectory at each bus during the simulation period, the action characteristics of the station layer out-of-step protection are shown in Figure 12.

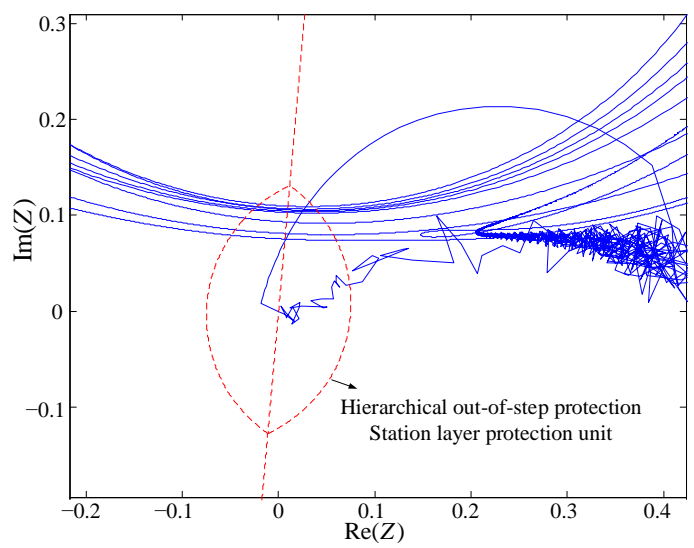

(a)

Figure 12. Cont. 


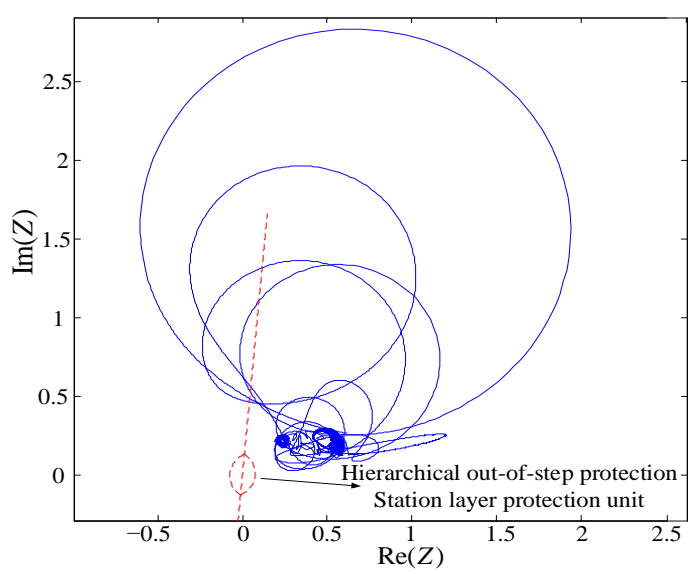

(b)

Figure 12. Measured impedance trajectory and the station layer protection action characteristics at each bus: (a) measured impedance trajectory at M1 and the station layer out-of-step protection action characteristics and (b) measured impedance trajectory at M2 and the station layer out-of-step protection action characteristics.

In Figure 12, the measured impedance trajectory at M1 can stably enter the action area of the station layer out-of-step protection. In order to verify the judgment of the station layer out-of-step protection, the local layer action areas of the generators G1 and G2 are defined as the multi-generators out-of-step mode. However, since the measured impedance trajectory at M2 does not enter the action area of the station layer protection, it can be judged that the equivalent generator G3 is only synchronous oscillation. Therefore, the station layer judges that there is not the multi-generators out-of-step fault at M2, so the local layer action area of G3 is defined according to the single generator out-of-step mode, which is the same as the existing out-of-step protection action area. The impedance trajectory measured at the terminal of each equivalent generator and the corresponding out-of-step protection action characteristics are shown in Figure 13.

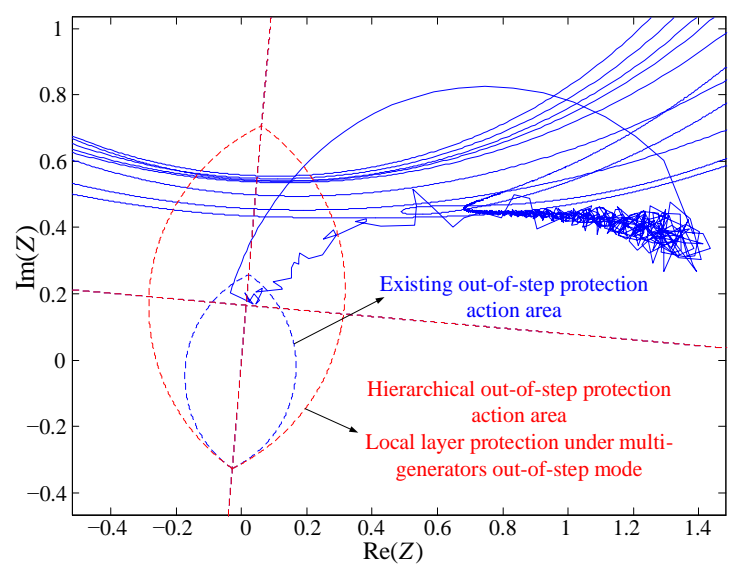

(a)

Figure 13. Cont. 


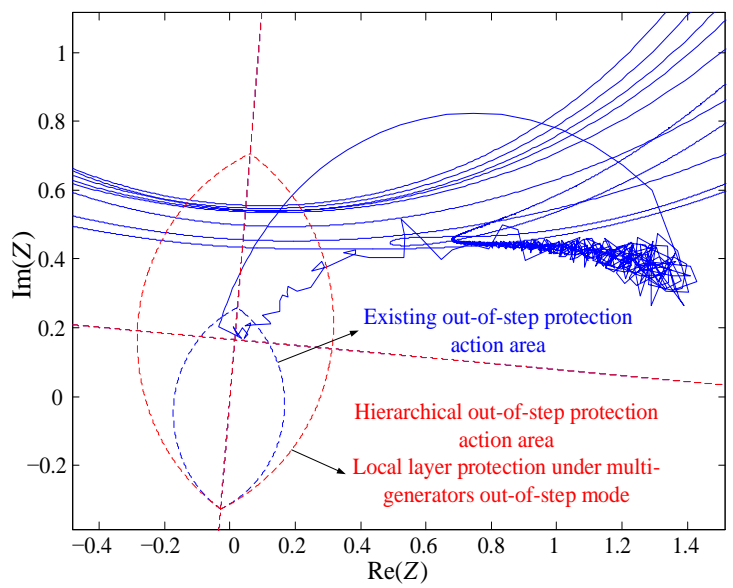

(b)

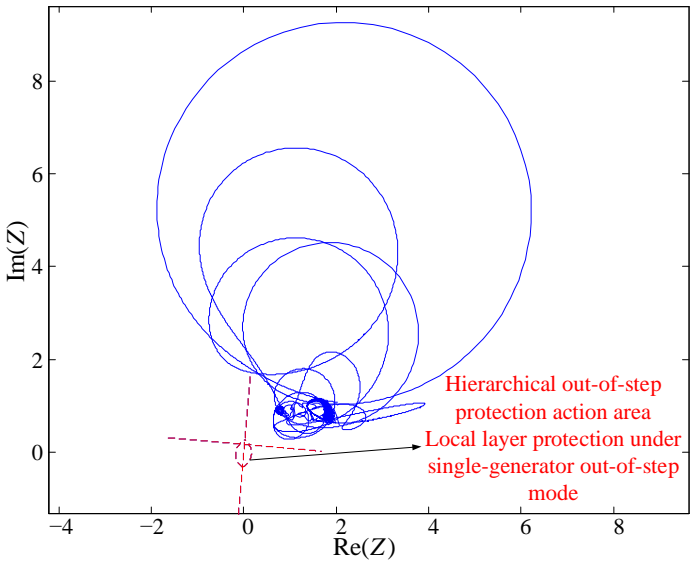

(c)

Figure 13. Measured impedance trajectory and the local layer protection action characteristics: (a) measured impedance trajectory of G1 and the local layer out-of-step protection action characteristics, (b) measured impedance trajectory of G2 and the local layer out-of-step protection action characteristics and (c) measured impedance trajectory of G3 and the local layer out-of-step protection action characteristics.

In Figure 13, the red dotted line represents the action characteristic of the proposed hierarchical local layer out-of-step protection and the blue dotted line represents the action characteristic of the existing out-of-step protection. According to the simulation results in Figure 13a,b, the measured impedance trajectory of the out-of-step generator only enters the action area of the existing out-of-step protection action characteristic within a short time, and the impedance trajectory of the stable out-of-step oscillation is completely outside the action area. Thus, the existing out-of-step protection refuses to act. Since the hierarchical out-of-step protection has considered the increasing effect of the oscillating current on the system impedance consisted by other generators, the protection action area has been adjusted. The simulation results in Figure 13a,b show that the multi-generators connected at one bus out-of-step fault can be accurately identified, and the crossing time of the measured impedance trajectory meets the requirements. In Figure 13c, since station layer has judged that the equivalent generator G3 is only synchronous oscillation, the protection action characteristics of the hierarchical local layer out-of-step protection and the existing out-ofstep protection are the same. The simulation results show that the measured impedance trajectory does not enter the action area, and the protection will not act.

Hierarchical out-of-step protection can realize the coordination between the station and local layer protection through real-time information interaction between different layers and can effectively solve the problem that the existing protection scheme cannot 
accomplish the identification of multi-generators connected in one bus out-of-step fault conditions. The protection area boundary can be adaptively adjusted according to the actual operation mode of the system and the power plant, so that the protection criterion can adapt to the real-time operating condition. In addition, by fusing the protection information from the stability control system of the grid-side, a reasonable generator tripping strategy can be constructed to guarantee the safe operation of the generators and the power grid.

\section{Key Problems of the Hierarchical Power Generation-Side Protection System}

There are many problems which need to be studied to construct a complete multiinformation fusion-based hierarchical power generation-side protection system. The key problems include the following aspects shown in Figure 14.

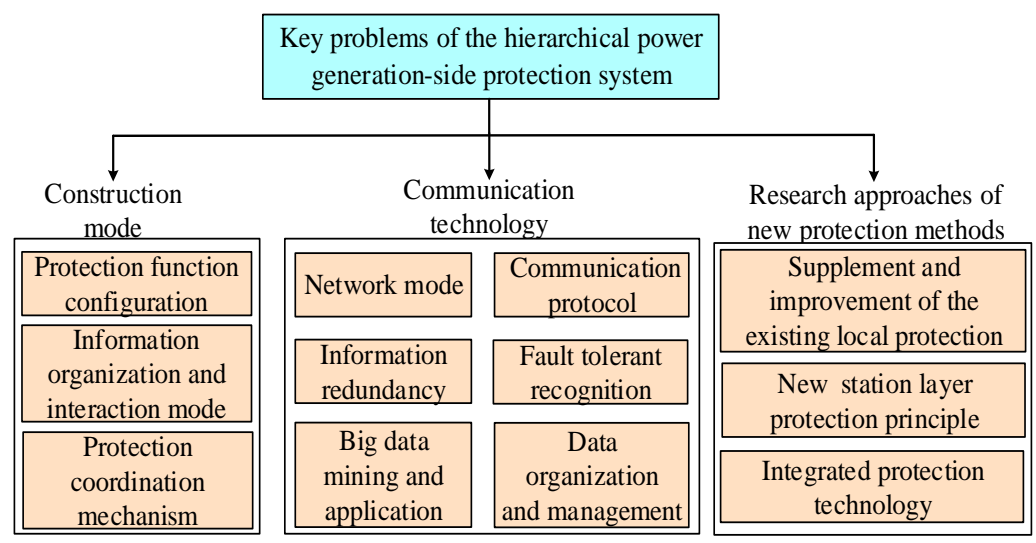

Figure 14. Key problems of the hierarchical power generation-side protection system.

\subsection{Construction Mode of the Hierarchical Power Generation-Side Protection System}

The local and station layer protection function should be determined based on the security and coordinated operation requirements of the protection performance and the information acquisition requirements of the hierarchical protection system. In order to determine the information organization and interaction mode among the hierarchical protection layers between generation units and between the generation and grid sides, the information from the CMS and the protection layers need to be analyzed. Moreover, the coordination mechanism between the station layer protection and the local layer protection needs to be studied.

\subsection{Communication Technology of the Hierarchical Power Generation-Side Protection System}

According to the data interaction requirements of the hierarchical protection system, the communication network mode and the data protocol need to be designed. In addition, the communication interaction method between the system generation and grid sides needs to be determined. The information redundancy and fault tolerant identification technology need to be studied to analyze the reliability of the communication system. Furthermore, the big data technologies need to be studied such as information mining and application, data organization and management, etc.

\subsection{Research Approaches of New Hierarchical Power Generation-Side Protection Methods}

The multi-information fusion-based hierarchical protection system opens a new way to improve the power generation-side protection. The research of the new hierarchical protection power generation-side protection methods can be started from the following aspects:

(1) Research on the supplement and improvement of the existing protection principle. This includes the improvement of the existing local protection and grid-related protection. The improved protection principle should fully adapt to the protection requirements under the complex power network operating environment. 
(2) Research on the station layer protection based on the collaborative operation between the power generation and grid sides. This kind of research is rich in content, including the safe cooperated operation between the generation and grid sides, and making full use of the sufficient information support from the hierarchical protection system, etc. The protection should attain the purpose of serving the safe and stable operation of the power system, so as to ensure the cooperation and interaction between the two system sides. On the premise of meeting the healthy condition of the generation-side units and the regulation demand of the power grid, the effective coordination of the protection action strategies should be realized.

(3) Research on comprehensive protection technology to ensure the efficient operation of the power generation and grid sides. Based on multi-information sources, the health conditions of the generation units and the safety restrictions of the power grid can be reflected. Through reasonable cooperation between the hierarchical layers, the generation control should be accomplished under real-time security constraints.

\section{Conclusions}

In order to improve the generation-side security and the coordination performance between the power system generation and grid sides, the existing power generationside protection mode is improved through the idea of a multi-information fusion-based hierarchical protection system.

(1) This paper presents a multi-information fusion-based hierarchical generation-side protection system. The system layer provides the system dispatch and control information to enable the generation-side to master the operation condition and dispatching demand of the system. The station layer realizes the information interaction among the generation units, the power grid and the automatic monitoring system. The local layer integrates abundant protection and control information to improve the protection performance.

(2) In view of the existing protection problems, hierarchical protection methods are constructed based on the multi-information fusion-based comprehensive local layer protection method and the multi-generators information fusion-based hierarchical protection method. Case analysis proved that, compared with the existing protection methods, the hierarchical single transverse differential protection comprehensive criterion can effectively enhance the protection sensitivity under the generator internal faults. The hierarchical out-of-step protection can adaptively adjust the protection action characteristic and generator tripping strategy according to the system operating condition and other generators' fault conditions, which can provide reliable guarantee for the safe operation of the generation and grid sides.

(3) The proposed protection methods are built on the basis of a perfect communication network, and the effect of information distortion is not considered. In order to provide reference and inspiration for the follow-up research, this paper discusses the key problems of the multi-information fusion-based hierarchical generation-side protection system which need to be further studied.

Author Contributions: Conceptualization, X.Y. (Xianggen Yin), Y.W. and J.Q.; data curation, X.Y. (Xianggen Yin), Y.W., J.Q. and X.Y. (Xin Yin); Formal analysis, X.Y. (Xianggen Yin); funding acquisition, X.Y. (Xianggen Yin); investigation, Y.W., J.Q., W.X. (Wen Xu), X.Y. (Xin Yin) and L.J.; methodology, X.Y. (Xianggen Yin) and Y.W.; resources, X.Y. (Xianggen Yin), Y.W., W.X. (Wen Xu), X.Y. (Xin Yin) and L.J.; validation, J.Q. and W.X. (Wen Xu); writing—original draft, X.Y. (Xianggen Yin), Y.W. and J.Q.; writing-review and editing, Y.W., W.X. (Wen Xu), L.J. and W.X. (Wei Xi). All authors have read and agreed to the published version of the manuscript.

Funding: This work was supported by the key project of smart grid technology and equipment of national key research and development plan of China under Grant 2016YFB0900600.

Institutional Review Board Statement: Not applicable. 
Informed Consent Statement: Not applicable.

Data Availability Statement: Data is contained within the article.

Conflicts of Interest: The authors declare no conflict of interest.

\section{References}

1. Technical Report on the Events of 9 August 2019. Available online: https://www.nationalgrideso.com/document/152346 /download (accessed on 1 August 2020).

2. Appendices to the Technical Report on the Events of 9 August 2019. Available online: https://www.nationalgrideso.com/ document/152351/download (accessed on 5 August 2020).

3. Huadong, S.; Tao, X.; Qiang, G.; Yalou, L.; Weifang, L.; Jun, Y.; Wenfeng, L. Analysis on Blackout in Great Britain Power Grid on 9 August 2019 and Its Enlightenment to Power Grid in China. Proc. CSEE 2019, 39, 6183-6192.

4. Project Group Turkey. Report on Blackout in Turkey on 31 March 2015; ENTSO-E: Brussels, Belgium, 2015.

5. Yao, S.; Yong, T.; Jun, Y.; Ansi, W. Analysis and lessons of Blackout in Turkey Power Grid on 31 March 2015. Autom. Electr. Power Syst. 2016, 40, 9-14.

6. Weifang, L.; Jun, Y.; Qiang, G.; Zhiwen, W.; Qi, J.; Fangfang, Y. Analysis on Blackout in Argentine Power Grid on 16 June 2019 and Its Enlightenment to Power Grid in China. Proc. CSEE 2020, 40, 2835-2842.

7. Jiandong, Y.; Kun, Z.; Ling, L.; Peng, W. Analysis on the causes of units 7 and 9 accidents at Sayano-Shushenskaya hydropower station. J. Hydroelectr. Eng. 2011, 4, 226-234.

8. Razzaghi, R.; Davarpanah, M.; Sanaye-Pasand, M. A Novel Protective Scheme to Protect Small-Scale Synchronous Generators Against Transient Instability. IEEE Trans. Ind. Electron. 2013, 60, 1659-1667. [CrossRef]

9. Paudyal, S.; Ramakrishna, G.; Sachdev, M.S. Application of Equal Area Criterion Conditions in the Time Domain for Out-of-Step Protection. IEEE Trans. Power Deliv. 2010, 25, 600-609. [CrossRef]

10. Wang, Y.; Yin, X.; Zhang, Z. The Fault-Current-Based Protection Scheme and Location Algorithm for Stator Ground Fault of a Large Generato. IEEE Trans. Energy Convers. 2013, 28, 871-879. [CrossRef]

11. Guilin, L.; Wei, S.; Xinli, S. Research on coordination of low excitation limit, loss of excitation protection and out-of-step protection based on grid-related protection. Power Syst. Prot. Control 2014, 42, 107-112.

12. Yang, Q.; Tang, X.; Su, Y.; Xiang, L.; Ding, M.; Mei, Y. An Optimal Allocation Scheme for Relay Setting Related to Power Grid and Over Frequency Generator Tripping Measures. Autom. Electr. Power Syst. 2013, 37, 127-131+135.

13. Phadke, A.G. Synchronized phasor measurements in power systems. IEEE Comput. Appl. Power 1993, 6, 10-15. [CrossRef]

14. Lee, G.; Kim, D.-I.; Kim, S.H.; Shin, Y.-J. Multiscale PMU Data Compression via Density-Based WAMS Clustering Analysis. Energies 2019, 12, 617. [CrossRef]

15. Bagher, M.; Jabali, A.; Kazemi, M.H. Power System Event Ranking Using a New Linear Parameter-Varying Modeling with a Wide Area Measurement System-Based Approach. Energies 2017, 10, 1088.

16. Serizawa, Y.; Myoujin, M.; Kitamura, K.; Sugaya, N.; Hori, M.; Takeuchi, A.; Shuto, I.; Inukai, M. Wide-area current differential backup protection employing broadband communications and time transfer systems. IEEE Trans. Power Deliv. 1998, 13, $1046-1052$. [CrossRef]

17. Serizawa, Y.; Imamura, H.; Kitamura, K. Performance evaluation of IP based relay communications for wide area protection employing external time synchronization. In Proceedings of the 2001 IEEE Power Engineering Society Summer Meeting, Vancouver, BC, CA, 15-19 July 2001; pp. 909-914.

18. Tan, J.C.; Crossley, P.A.; Kirschen, D.; Goody, J.; Downes, J.A. An expert system for the back-up protection of a transmission network. IEEE Trans. Power Deliv. 2000, 15, 508-514. [CrossRef]

19. Shalini; Samantaray, S.R.; Sharma, A. Enhancing Performance of Wide-Area Back-Up Protection Scheme Using PMU Assisted Dynamic State Estimator. IEEE Trans. Smart Grid 2019, 10, 5066-5074. [CrossRef]

20. Jena, M.K.; Samantaray, S.R.; Panigrahi, B.K. A New Adaptive Dependability-Security Approach to Enhance Wide Area Back-Up Protection of Transmission System. IEEE Trans. Smart Grid 2018, 9, 6378-6386. [CrossRef]

21. He, J.; Zhu, G.; Bo, Z. Integrated Protection for Power Systems Based on the Multi-Agent Technology. Trans. China Electrotech. Soc. 2007, 6, 141-147.

22. Bo, Z.Q.; He, J.H.; Dong, X.Z.; Caunce, B.R.J.; Klimek, A. A multi-algorithm based integrated protection scheme for distribution systems. In Proceedings of the 7th Institution of Engineering and Technology International Conference on Advances in Power System Control, Operation and Management (APSCOM 2006), Hong Kong, China, 30 October-2 November 2006.

23. Weijian, W. Electrical Equipments Relaying Protection Principle and Application, 2nd ed.; Electric Power Press: Beijing, China, 2001.

24. Cui, X.; Tai, N.; Liu, J. New Unit-transverse Differential Protection Based on the Fault Contributed Zero Sequence Current. Autom. Electr. Power Syst. 2007, 12, 61-63.

25. Zhang, K. Protection System and New Dynamic Simulation Experiment Technology of Huge Hydro Generator; Huazhong University of Science and Technology: Wuhan, China, 2008.

26. Zhang, K.; Yin, X.; Chen, D.; Zhang, Z. Dynamic Simulation Experiments for Internal Fault Protection of Large Hydro-generators. Autom. Electr. Power Syst. 2008, 6, 85-90. 
27. Yang, J. Study on Fault Transient Simulation for Huge Hydro-Generators and Protection Technology for Generator-Transformer Units; Huazhong University of Science and Technology: Wuhan, China, 2004.

28. Xia, Y. Study and Application on Huge Hydro Generator Internal Fault Transient Simulation and Optimized Main Protection Scheme; Huazhong University of Science and Technology: Wuhan, China, 2006.

29. Anderson, P.M. Protective Schemes for Stability Enhancement, in Power System Protection; IEEE Press: New York, NY, USA, 1998.

30. Reimert, D. "Loss of Synchronism" Protective Relaying for Power Generation Systems; CRC Press Taylor \& Francis Group: New York, NY, USA, 2006. 